\title{
Text, Visualisierung und Erinnerungskultur. Indo-Islamische Bauinschriften als mnemotechnische, sinnstiftende Medien eines kollektiven historischen Denkens
}

Der vorliegende Beitrag wird am Beispiel der Bauinschriften des Quwwat al-Islām ${ }^{1}$ Komplexes in Delhi aus dem 12.-14. Jahrhundert die Frage diskutieren, ${ }^{2}$ welche Ergebnisse sich auf der Basis eines erweiterten Textverständnisses über ihre ,lesbare‘ textliche Aussage hinaus von diesen Objekten ableiten lassen. Ein besonderes Augenmerk gilt hierbei der Frage, welche Inhalte auf welche Weise und mit welcher Intention durch dieses vielschichtige Medium transportiert wurden.

Das unter dem Namen Quwwat al-Islām-Moschee oder Quṭb-Komplex bekannte Gebäudeensemble befindet sich im Süden von New Delhi im heutigen Stadtteil Merauli, ca. $13 \mathrm{~km}$ vom zentralen Connaught Place entfernt. ${ }^{3}$ Bei dem Gebäude handelt es sich um die bedeutendste Freitagsmoschee der ersten muslimischen politischen Machtelite, die sich Ende des 12. Jahrhunderts in Nordindien etablieren konnte. In der öffentlichen Wahrnehmung und Darstellung, aber auch von einem Großteil der indischen Forschung nach der Unabhängigkeit zumeist auf der Basis der Forschungsarbeiten aus der Kolonialzeit, wird die Moschee - nicht zuletzt auf Grund der Aussagen, die aus den Inschriften abgeleitet werden, als visualisierter Markstein eines historischen, mit Gewalt herbeigeführten Bruchs in der indischen Geschichte und als Sinnbild für den Beginn der muslimischen Fremdherrschaft auf dem indischen Subkontinent angesehen. ${ }^{4}$

1 Im Folgenden wird eine modifizierte Umschrift der Deutschen Morgenländischen Gesellschaft (DMG) verwendet. Auf Assimilationen und grammatikalische Flexionen wird verzichtet, da neben Quellen in Arabisch, Persisch, Urdu und ihren Mischformen auch Sanskrit und weitere indische Sprachen in der Forschung zur frühen indo-islamischen Forschung herangezogen werden müssen.

2 Dieser Beitrag basiert auf Teilen meiner Forschungsarbeit zum Quṭb-Komplex. Die Ergebnisse dieser Arbeit werden in Kürze publiziert. Siehe Redlinger 2012.

3 Trotz der großen kunstgeschichtlichen Bedeutung dieses Gebäudes existieren bisher keine umfassenden Bauaufnahmen und detaillierten archäologischen Berichte. Die meisten der größtenteils unvollständigen Beschreibungen der Moschee stammen aus der britischen Kolonialzeit. Siehe hierzu Page 1926, Cole 1872, Cunningham/Beglar/Carlleyle 1874, 1-91 und Sanderson 1916, 120f. Zur Problematik der mangelhaften Dokumentation des Baus und seiner Rezeption in der Forschung siehe Redlinger 2012.

4 Siehe hierzu u. a. Kumar 2008, Mujeeb 2008 und Flood 2008. Zur Zerstörung der nicht-muslimischen Architektur durch die gūuridischen Invasoren und zur Wiederverwendung des Materials zum Bau von Moscheen in Indien im 12.-13. Jahrhundert siehe Eaton 2000, Patel 2009, Flood 2003. In seinen Arbeiten zur indischen Sultanatszeit zeigt Flood, dass dieses negative Bild der muslimischen Eroberer maßgeblich während der Kolonialzeit entstand, siehe hierzu besonders Flood 2007. Siehe

○ Open Access. (C 2018 Daniel Redlinger, publiziert von De Gruyter. (cc) BY-NC-ND Dieses Werk ist lizenziert unter der Creative Commons Attribution-NonCommercial-NoDerivatives 4.0 Lizenz.

https://doi.org/10.1515/9783110619928-012 
Eine herausragende Bedeutung für die Untersuchung des frühen Delhi-Sultanats im 12.-14. Jahrhundert erhält die Moschee, da drei bedeutende Herrscher in dem genannten Zeitraum diese Moschee als Medium des Ausdrucks für ihr jeweiliges Herrschaftsprogramm nutzten. So lässt sich anhand der Bauinschriften belegen, dass die Moschee unter Quṭb al-Dīn Aybak (r. 1206-1210) errichtet und von Šams al-Dīn Iltutmiš (r. 1211-1236) und 'Alā' al-Dīn Hilǧ̄i (r. 1296-1316) umgebaut und erweitert wurde. Angesichts der Tatsache, dass substantielle Schriftquellen aus diesem Zeitraum fehlen, die über die Rezeptions- und Konzeptionsgeschichte des Baus berichten, ${ }^{5}$ bietet die Analyse der Architektur, des Dekors und besonders der Inschriften ${ }^{6}$ der Moschee als Stein gewordene nonverbale herrschaftspolitische Kommunikation und somit als Teil des Prozesses einer Gesellschaftsbildung eine einzigartige Möglichkeit, Einblick in die sich wandelnden Strategien politischer Selbstinszenierung zu erhalten. ${ }^{7}$ Besonders der Bestand der Bauinschriften am Quṭb-Komplex, der zu den umfangreichsten im Wirkungsraum islamisierter Gesellschaften der Vormoderne gehört, ${ }^{8}$ verschafft uns hier vielschichtige Einsichten.

Im Bereich der Islamischen Kunstgeschichte und angrenzender Disziplinen, die sich mit der Architektur der islamisierten Welt der Vormoderne beschäftigen, werden Bauinschriften - weit mehr als der geometrischen Dekor in der Architektur - als

zur Problematik und Genese dieses negativen Blicks auf die muslimische Herrschaft in Indien im 12.-14. Jahrhundert, sowie die Verwendung des Quṭb-Komplexes als Referenzmöglichkeit für diese politisch motivierten Geschichtsnarrative Redlinger 2012.

5 Zur Problematik der schriftlichen Quellen insbesondere der Historiographien aus dem 12.-18. Jahrhundert in Indien als Möglichkeit der Rekonstruktion ,realer' historischer Prozesse siehe Conermann 2002, 20-22.

6 Ein Teil der Inschriften im Quṭb-Komplex wurde unter der Kolonialregierung von dem Archaeological Survey of India aufgenommen, siehe hierzu besonders Husain 1936, 96-119. Auf eine genaue Angabe der Lage der jeweiligen Inschriften wurde bei der Bearbeitung weitgehend verzichtet. Es ging den Bearbeitern der Inschriften vielmehr um eine Auflistung der Inhalte für weiterführende philologische und historische Studien. Die Übersetzungen der Inschriften sind oft ungenau und fehlerhaft. Die genaue Lokalisierung der Inschriften und eine vollständig neue Übersetzung dieser bildet einen Teil meiner Dissertation. Siehe Redlinger 2012. Die Gesamtzahl der Bauinschriften im Quṭb Komplex liegt weit über 200. Sie umfasst neben kurzen religiösen Formulae vor allem längere Koranpassagen, hadițe, Eulogien und Bauangaben. Die Sprachen der Inschriften sind Persisch, Arabisch und Mischformen aus beiden.

7 Der Ansatz, materielle Kultur als Teil eines Kommunikationssystems zu verstehen, wurde für den indo-islamischen Kontext im Besonderen von Flood 2007 und 2009 verfolgt.

8 Besonders im sogenannten indo-islamischen Kontext fällt es schwer, die Bezeichnung, islamisch für eine gesellschaftliche, politische und religiöse Kategorie zu verwenden. Im Folgenden wird daher weitgehend der Begriff ,islamisiert‘ an Stelle von ,islamisch“ verwendet um auszudrücken, dass die behandelten Gesellschaften, Prozesse, Objekte und Kulturtechniken zwar auch, jedoch nicht ausschließlich durch diesen Glauben geprägt wurden. Eine ähnliche Problematik ergibt sich auch im mamlukischen Ägypten bis zum Ende des 14. Jahrhunderts mit seinem großen koptischen Bevölkerungsanteil. Siehe zur Islamisierung der koptischen Bevölkerung O’Sullivan 2006. 
das prägnanteste künstlerische Ausdrucksmittel einer durch ,den Islam`9 geprägten Gesellschaft verstanden. ${ }^{10}$ Trotz dieser den Bauinschriften zugesprochenen Bedeutung wird der spezifische Charakter der arabischen Schrift und Sprache sowie der durch sie beeinflussten Sprachen und Schriften, wie Persisch, Urdu als visuelles und textliches Medium der Kommunikation innerhalb eines architektonischen Raumes nur in wenigen Fällen erkannt und für eine Untersuchung berücksichtigt. ${ }^{11}$ In vielen Fällen beschränkt sich die Auseinandersetzung mit Inschriften auf die Zuordnung ihrer kalligraphischen Form ${ }^{12}$ und die Extraktion von benötigten inhaltlichen Informationen zur Kontextualisierung des Baus oder historischer Ereignisse. Dieses auf die Textinhalte begrenzte Verfahren bei der Untersuchung von Bauinschriften findet auch bei den arabischen und persischen Bauinschriften des Delhi-Sultanats Anwendung; in diesem Fall dienen die Inschriften mehrheitlich dazu, ein weitgehend negativ konnotiertes Geschichtsbild zu stützen. In den Arbeiten zu Inschriften am Quṭb-Komplex werden höchst selektiv Textausschnitte, wie prominente Suren oder Teile von Herrscherinschriften, aus dem Gesamtzusammenhang der Inschriftensysteme des QuṭKomplexes gelöst und diese Textausschnitte als Belege zu Interpretationen von historischen Ereignissen einschließlich von Herrschaftsstrukturen herangezogen. ${ }^{13}$

Diese selektiv ausgerichtete Verwendung von Bauinschriften als Beleg für historische Ereignisse spiegelt ein Text- und Schriftverständnis wider, das im Umgang mit nichtsemitischen Schriftsystemen geschult wurde. ${ }^{14}$ Einen positiven Impuls für die kritische Revision der zum Teil im 19. Jahrhundert entwickelten methodischen Herangehensweisen an Texte erhielt die kunstgeschichtliche, historische und philologische Forschung zu außereuropäischen Kulturen in jüngster Zeit in Deutschland durch die

9 Zur Problematik des Begriffs ,Islam‘ als kulturelles, soziales und künstlerisches Determinierungsmuster siehe Grabar 2006.

10 Siehe hierzu Welch 1977, der anhand eines Vergleichs zur Schriftkultur des Christen- und Judentums die Frage diskutiert, welche Funktion Schrift ,im Islam“ besitzt. Siehe zur Funktion und Bedeutung der Bauinschriften unter den Mamluken in Ägypten Juvin 2013 und im abbasidischen Kontext Tabbaa 2001, 25.

11 Ein gutes Gegenbeispiel liefert Harithy 2010 in einem Beitrag über die Funktion der Inschriften im mamlukischen Kairo.

12 Besonders offensichtlich wird das eurozentrische Verständnis von Text und Schrift in dem Standardwerk zur Islamischen Kalligraphie von Blair durch ihre Ableitung des Begriffs Kalligraphie von nichtsemitischen Schriftsystemen (Blair 2006, xxv, 3-7). Siehe hierzu auch das zweite Kapitel „The Intermediary of Writing“ in Grabar 1992, 47-117.

13 Siehe zu den Inschriften des Quț-Komplexes vor allem die Arbeiten von Welch 1985, Welch/Kushani/Bain 2002. Ein weiteres Beispiel für diese Form selektiver Auswahl in Flood 2009, 227-259.

14 Teile von inhaltlichen Aussagen der Bauinschriften aus der frühen Sulțānatszeit in Indien im 12.-14. Jahrhundert werden häufig als zusätzlicher Beleg für ,historische Fakten' herangezogen, die zumeist aus einer unkritischen Auseinandersetzung mit den nicht unproblematischen schriftlichen Quellen, wie Historiographien, generiert wurden. Siehe hierzu Redlinger 2012 und Fußnote 5. 
zunehmende methodische Einflussnahme der Kulturwissenschaften. ${ }^{15}$ Eine Diskussion dieser kulturwissenschaftlichen Überlegungen in Disziplinen wie den Sprachwissenschaften steht jedoch, zumindest soweit es den Umgang mit Bauinschriften aus islamisierten Kulturen betrifft, noch weitgehend aus. ${ }^{16}$

Für die Untersuchung der Bauinschrift am Quṭb-Komplex erwies es sich ertragreich, einige kulturwissenschaftliche methodische Überlegungen in die Analyse miteinzubeziehen. Ein wichtiger Punkt war dabei das Überdenken des Text- und Schriftverständnisses sowie der herkömmlichen Vorstellungen von der ,Funktionsweise“ der Bauinschriften. Hiermit verbunden war die Frage, inwieweit sich der Text der Inschriften überhaupt übersetzen und in Folge als historische Quelle interpretieren lässt, wenn er nicht nur als ,Steinbruch“ für die Rekonstruktion historischer Ereignisse dienen soll. ${ }^{17}$ Einen sehr ertragreichen Ansatz liefert hier die Untersuchung von Krämer über die ,Schriftbildlichkeit‘ geschriebener Texte, in der die Autorin Schrift über ihre zweidimensionale Verwendung im Raum hinaus um weitere Dimensionen der Rezeption erweitert. ${ }^{18}$ Einen weiteren wichtigen Schritt hin zum erweiterten Verständnis von Bauinschriften liefert Hilgert mit seinem Ansatz der ,TextAnthropologie', nach der Schriftquellen als „Netz sozialer (Rezeptions-)Praktiken, in das das Geschriebene als artefaktisches ,Objekt' und ,Repräsentation“ [...] epistemischen Handelns eingebunden ist“" verstanden werden müssen. ${ }^{19}$ Nach Hilgert muss der „kulturwissenschaftliche Interpret [...] sich also um eine ,Kartierung“ der rezeptionspraktischen ,Text-Akteur-Relationen', die als eine Form von ,Subjekt-ObjektNetzwerken“ in den ,Natur/Kultur-Geweben“ des Sozialen verortet sind“ bemühen. ${ }^{20}$ Diese erweiterte Untersuchung der „Materialität und Präsenz des Geschriebenen“²1 innerhalb eines architektonischen Raumes erweitert den Blick auf die Inschriften um die Analyse der Rezeptionspraktiken und die mit ihnen verbundenen Kognitionsprozesse. Eine solche Herangehensweise setzt jedoch voraus, dass Architektur selber

15 Einen guten Überblick über mögliche Perspektiven in der kunsthistorischen Forschung liefert zum Beispiel der Ausstellungsband Lermer/Schalem 2010 und die 6. Ausgabe der Zeitschrift Journal of Art Historiography von 2012.

16 Angesichts des euphorisch gefeierten kulturwissenschaftlichen und methodischen ,awakening in den ,kleinen Disziplinen in Deutschland wird jedoch gerne vergessen, dass die systematische und umfassende Erschließung und Deutung eines (vormodernen) Artefakts erst auf der Grundlage einer fundierten, kulturspezifischen, philologischen und kunsthistorischen Ausbildung möglich ist. Für weitere Überlegungen über Möglichkeiten der Einbeziehung der kulturwissenschaftlichen Ansätze im Bereich einer außereuropäischen Kunstgeschichte siehe Redlinger 2012a.

$17 \mathrm{Zu}$ den Problemen der Verwendung von historischen Texten als ,Schlüssel` zur Rekonstruktion vergangener Lebenswirklichkeit siehe Hilgert 2010, 1f.

18 Krämer 2003, 159.

19 Hilgert 2010, $92 \mathrm{f}$.

20 Hilgert 2010, 93.

21 Hilgert 2010, 98-104. 
als ,Text' verstanden wird und die ,Intertextualität‘ - also die Summe der in ihr in Erscheinung tretenden Ausdrucksformen des ,Textes' - umfassend untersucht wird. ${ }^{22}$

Für die Bauinschriften am Quṭb-Komplex bedeutete dies konkret, die Analyse der Bauinschriften nicht auf deren rein lesbare Aussage zu beschränken, sondern darüber hinaus die Positionierung der Inschriften im architektonischen Raum, die Verwendung der unterschiedlichen Textgattungen, die Wahl der Sprache, die kalligraphische Gestaltung als Teil eines ästhetischen Ausdrucks sowie das Verhältnis der Schrift zu anderen Dekorelementen miteinander in Bezug zu setzen. So ist für die Analyse der Inschriften am Quṭb-Komplex die Beobachtung wichtig, dass eine Vielzahl der Inschriften für einen Besucher der Moschee im 12.-14. Jahrhundert auf Grund ihrer Lage oder Form nur schwer oder gar nicht lesbar waren. Folgt man Cruikshank Dodds und Khairallahs Konzept eines „Image of the Word“23, hilft hier der Hinweis, dass Inschriften in islamisierten Regionen innerhalb eines völlig anderen Konzepts von Ikonographie existieren. Durch die bloße Präsenz des Arabischen manifestiert sich in ihnen das Sinnbild des Wortes Gottes. Bauinschriften sind somit für muslimische Gläubige, die auf Grund ihrer religiösen Erziehung Zugang zu diesem exklusiven Kommunikationssystem haben, ein System von Verweisen auf religiöse Narrative. Besonders die religiösen Inhalte, die in den Inschriften einen großen Raum einnehmen, beziehen sich - maßgeblich auf orale Traditionen gestützt - auf eine komplexe Erfahrungs- und Gedankenwelt. ${ }^{24}$ Die Inschriften als künstlerische und dauerhafte Darstellungsform greifen somit auf eine Ästhetik des Erinnerns, der sozial determinierten Erfahrungen, der Assoziationen und auf individuelle Phantasievorstellungen zurück. Vergleichbar ist dieses Erfahren von Text mit den Beobachtungen Beltings im christlichen Kulturbereich, für den er zeigt, dass das Lesen von Text oder das Betrachten von Bildern mit einer eigenständigen Visualisierung der Themenbereiche im Kopf des Rezipienten verbunden ist. ${ }^{25}$ Im Folgenden werden an einigen ausgewählten Beispielen aus dem Quṭb-Komplex verschiedene Formen und Konzepte dieser Translationsprozesse diskutiert.

Bereits seit dem 7./8. Jahrhundert existierten kleine muslimische Gemeinden und Gemeinschaften von Händlern an den Küsten und im Innern des indischen Subkontinents. ${ }^{26}$ Bis zur Invasion Indiens durch muslimische Heere im 13. Jahrhundert etablierte sich dort jedoch dauerhaft kein größeres Herrschaftssystem mit einer islamisierten Elite. Nordindien war lediglich Ziel saisonal begrenzter Beutezüge von muslimischen Herrschern aus den westlich vom Subkontinent gelegenen Grenzregionen.

22 Siehe zur Idee, Architektur als Text zu verstehen, Gomolla 2002.

23 Siehe hierzu Cruikshank Dodd 1969 und Cruikshank Dodd/Khairallah 1981.

24 Siehe zur Ver- und Übermittlung von Wissen in islamisierten Gesellschaften der Vormoderne am Beispiel des mamlukischen Kairo Berkey 1992.

25 Siehe hierzu Belting 2006 und 2012.

26 Siehe zu den muslimischen Gemeinden vor und während der Eroberung des Subkontinents durch gūuridische Heere Patel 2004, 2004 a und 2004b; Shokoohy 1988, 1993 und 2003. 
In den muslimischen Quellen jener Zeit werden diese Raubzüge als ġazwa, religiös legitimierte Angriffskriege, verklärt. ${ }^{27}$

Im Zuge der Eroberung des indischen Subkontinentes durch die Ġūriden kam es zur Etablierung von Herrschaftssystemen, in denen in großen Teilen Nordindiens eine islamisierte Elite als Minorität über eine mehrheitlich nichtmuslimische Bevölkerung herrschte. ${ }^{28}$ Dass diese Einrichtung eines durch den Islam legitimierten Herrschaftssystems keinen plötzlichen Bruch in der indischen Geschichte darstellte, sondern dass die muslimischen Eliten sich bereits vor der Eroberung Nordindiens bei ihrer Herrschaftslegitimierung lokaler - auch nichtmuslimischer - Strategien bedienten, wurde in jüngster Zeit durch die Arbeiten von Flood gezeigt. ${ }^{29}$

Die neue Herrschaftselite Nordindiens sah sich mit einer Bevölkerung konfrontiert, die besonders in den ersten dreißig Jahren nach der Eroberung (1190-1220) in ihrer ethnischen, religiösen und sozialen Zusammensetzung sehr heterogen war. Angehörige der verschiedenen, teilweise erst spät islamisierten Stämme und Volksgruppen aus Zentralasien, dem östlichen Iran, dem heutigen Afghanistan und Pakistan bildeten neben lokalen nichtmuslimischen Fürsten nach der Eroberung des Subkontinents in den wichtigsten Herrschaftszentren wie Delhi die politischen Eliten. ${ }^{30}$ Neben dieser in sich heterogenen Führungsschicht standen bis ins letzte Drittel des 13. Jahrhunderts viele Militärsklaven ohne Stammesbindungen, die ausschließlich ihrem Herrn treu ergeben waren. Zu ihnen gehörten auch Quṭb al-Dīn Aybak, einer der ersten wichtigen Herrscher Nordindiens, ein ehemaliger ġūridischer Militärsklave (r. 1206-1210), und dessen eigener Militärsklave, ${ }^{31}$ Schwiegersohn und Nachfolger Šams al-Dīn Iltutmiš (r. 1211-1236).

Unter Iltutmiš als sulțān wurde Nordindien nach einem langen Ringen unter einer zentralen Herrschaft vereint. Er machte sich de facto von den Ġūriden unabhängig und ließ sich 1229 durch den Kalifen in Bagdad als sultān legitimieren. ${ }^{32}$ Nach dem Tod von Iltutmiš 1236 folgten ihm eine Reihe seiner Söhne und eine Tochter auf dem Thron. Die mit diesen Herrscherwechseln einhergehende Auflösung des indischen Reiches ereignete sich zeitgleich mit der Zerschlagung des abbasidischen Reiches und dem damit verbundenen Verlust der zentralen Autorität des Kalifats. Nicht zuletzt in Folge der Bedrohung durch die Mongolen strömte eine Vielzahl von Flüchtlingen aus

27 Siehe hierzu Flood 2001.

28 Siehe hierzu Wink 1997; Kumar 2010.

29 Flood 2009 zeigt an Hand des ausgedehnten physischen und geistigen Austauschs von unterschiedlichen Elementen der Objektkultur, wie diplomatischen Geschenken, Mode und Architektur, die Vielschichtigkeit der wechselhaften Einflüsse und Beziehungen im 8.-13. Jahrhundert in Regionen, die durch den Indischen Ozean miteinander verbunden waren.

30 Siehe hierzu Jackson 1999.

31 Zum Konzept der Militärsklaven am Hof in Delhi siehe Jackson 1990.

32 Hillenbrand 1988. 
den persischsprachigen Gebieten an den Hof in Delhi, wodurch auch die Hofetikette maßgeblich verändert wurde. ${ }^{33}$

1292 übernahm die Hilǧì-Dynastie die Herrschaft am Hof in Delhi. Besonders unter 'Alā' al-Dīn Hुilğī (r. 1296-1316) wurde Nordindien wieder unter einer zentralen Herrschaft vereint. ${ }^{34}$ Zudem konnte dieser Herrscher die Bedrohung durch die Mongolen endgültig abwehren. Unter seiner Regierung setzte sich die Persianisierung des Hofes durch den kontinuierlichen Zustrom von Gelehrten, Administratoren und Höflingen fort.

Eine Herausforderung für die neue politische Elite im 12.-14. Jahrhundert in Nordindien bestand auf Grund der wechselnden politischen sowie sozialen Herausforderungen und Prozesse maßgeblich darin, Wege zur Überbrückung der religiösen und ethnischen Heterogenität zu finden sowie eine allgemein verständliche ,Sprache` der verbalen und nonverbalen Kommunikation zu schaffen. Notwendig war dies auch, um Strategien zur Legitimierung ihres herrschaftlichen Selbstverständnisses zu entwickeln. Dies schloss den Rückgriff auf unterschiedliche identitätsstiftende Vergangenheiten mit ein. Hierbei mussten verschiedene Geschichtsnarrative in einer kohärenten Form zu einer gemeinsamen Erzähl- und Darstellungsform verknüpft werden. Eine Herausforderung war zudem auch die Reaktion auf überregionale Ereignisse; hierzu zählen die Veränderung des Verhältnisses zu den Gūriden und der Wegfall des abbasidischen Kalifats als übergeordneter, legitimierender Institution ebenso wie die Bedrohung durch die Mongolen und durch regionale muslimische und nichtmuslimische Fürsten. Die Herrschaftsarchitektur kann diesbezüglich auch als Spiegelbild für diese diplomatischen Prozesse dienen.

Unter Quṭb al-Dīn Aybak wurden um 1190 die erste Moschee auf den Resten einer Tempelanlage eines wichtigen Regionalfürsten und das erste Stockwerk eines Turmes, des sogenannten Quṭb Minār, errichtet. ${ }^{35}$ Ebenfalls während seiner Herrschaft wurde wenige Jahre später der Hauptgebetshalle eine Fassade vorgeblendet. Unter seinem Militärsklaven und Nachfolger Iltutmiš wurde die Moschee um 12301240 nach Norden, Süden und Osten erweitert sowie das Quṭb Minār um drei Stockwerke ausgebaut. Im Westen der Erweiterung wurde der Moschee im selben Zeitraum ein Grabbau angegliedert. Unter 'Alā' al-Dīn Hilğ̄ì wurde die Moschee 1310 nochmals um einen Gebetsraum im Norden und Kolonnaden im Norden, Osten und Süden vergrößert. Von dieser letzten Erweiterung sind jedoch außer einem Eingangstor, dem sogenannten 'Alā'-i Darwāza, nur noch Fundamente erhalten. ${ }^{36}$ Der umfangreiche, hier thematisierte Inschriftenbestand befindet sich in den Eingangsbereichen der ersten Moschee, auf den Blendfassaden vor den Hauptgebetsräumen der ersten

33 Zur Persianisierung am Hof in Delhi siehe Kumar 2009.

34 Niazi 1990.

35 Siehe dazu Page 1926, Anm. 1, 6-26, Pl. I-XII.

36 Auf Grund der Baureste ist davon auszugehen, dass die zweite Erweiterung niemals vollständig fertiggestellt wurde. Siehe auch dazu Page 1926, 15-17. 
Moschee und der Erweiterung des Iltutmiš, innen und außen am Grabbau, auf dem Quṭb Minār und auf dem 'Alā-i Darwāza.

Die unter Aybak errichtete erste Freitagsmoschee Delhis im Quwwat al-Islām Komplex folgt dem Raumkonzept einer Hofmoschee als Versammlungsbereich für die Gemeinde und als Ort für den Gläubigen, an dem dieser seinen religiösen Pflichten nachkommen kann. Visuell gestaltete man hingegen die wichtigsten architektonischen Elemente unter Rückgriff auf die Techniken des indischen Tempelbaus und unter Wiederverwendung des Baumaterials von zerstörten Tempeln (Abb. 1), die jeweils mehr oder weniger den Vorstellungen und Bedürfnissen muslimischer Idealtypen angepasst wurden. ${ }^{37}$ Die Kuppeln und Bögen basieren in der Herrschaftszeit Aybaks alle auf indischen Kragsteinkonstruktionen und entsprechen damit nur optisch, nicht aber technologisch ihren Vorbildern aus den westlich gelegenen islamisierten Kulturkreisen. Durch die Verwendung von Spolienmaterial aus nichtislamischen sakralen Gebäuden oder Spolienrepliken wurde eine optische Annäherung an die indische Tempelarchitektur erreicht. Die durch die Kürze der Säulen erzeugte geringe Raumhöhe im Hauptgebetsraum legt einen Vergleich zu der gedrungenen Raumwirkung im Sanktum eines Tempels nahe. Der Bezug zwischen der Raumwirkung des Hauptgebetsraumes und dem Sanktum eines Tempels lässt eine Übertragung von Vorstellungen in Bezug auf Raumhierarchien vermuten.

Eine Inschrift am Nordeingang, die besagt, dass die Moschee auf den Resten eines prestigeträchtigen religiösen nichtmuslimischen Gebäudes und mit dem Material von Tempeln aus der Umgebung errichtet wurde, ${ }^{38}$ stellt ebenfalls eine Verknüpfung von Moschee und vorislamischen religiösen Bauten her. Die bewusste (Wieder-)Verwendung von figürlichen Abbildungen wie z. B. kïrtimukha (sogenannte Ruhmesgesichter mit apotropäischer Wirkung), Planetendarstellungen, Tänzerinnen oder göttlichen Wesen sowie Sinn tragender Bauelemente aus der Tempelarchitektur wie den Gesimsformen zeigt, dass die ästhetische Wirkung und ihre Ikonographie in der Moschee ebenfalls mit einer bestimmten Absicht verwendet wurden. Besonders deutlich wird dies an einer persischen Bauinschrift mit der Nennung des Bauherrn Quṭb al-Dīn Aybak, die zusammen mit einem kīrtimukha gefertigt wurde (Abb. 2). Hier wurde der ursprüngliche, visuell verankerte Sinngehalt des Bildmotivs zusammen mit der textlichen und visuellen Aussage der Inschrift und ihrer Form in eine vielschichtige multimediale Aussage transformiert.

Einige Jahre nach der Fertigstellung der Moschee 1198/99 wurde durch die Vorblendung einer vollständig neu gestalteten Fassade mit einer großen Zahl von Inschriften und einem komplexen Dekor vor den Hauptgebetsraum das Aussehen des Gebäudes stark verändert (Abb. 3). Die Auswahl der ausschließlich in arabischer

37 Page 1926, Anm. 14, 6-10, Cunningham/Beglar/Carlleyle 1874, Anm. 1 und Flood 2009, Anm. 4, 137-226.

38 Page 1926, Anm. 14, 6 f. 


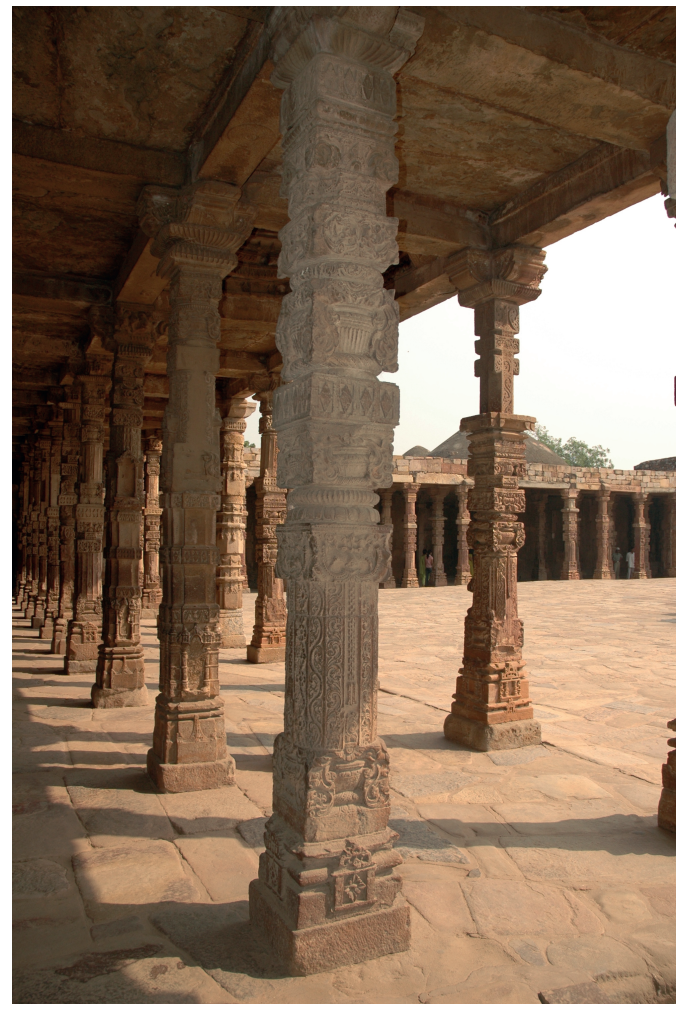

Abb. 1: Delhi, Quṭ-Komplex, Nordkolonnade der ersten Moschee, um 1200

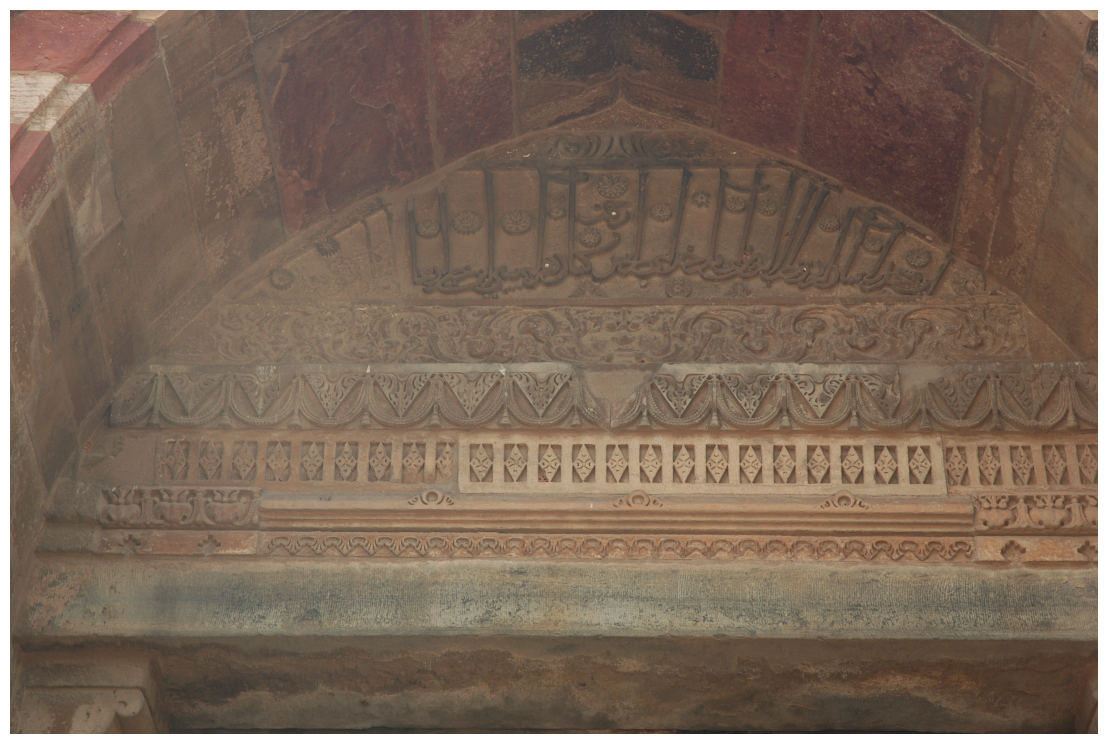

Abb. 2: Delhi, Quṭb-Komplex, Bauinschrift im Osteingang, um 1200 


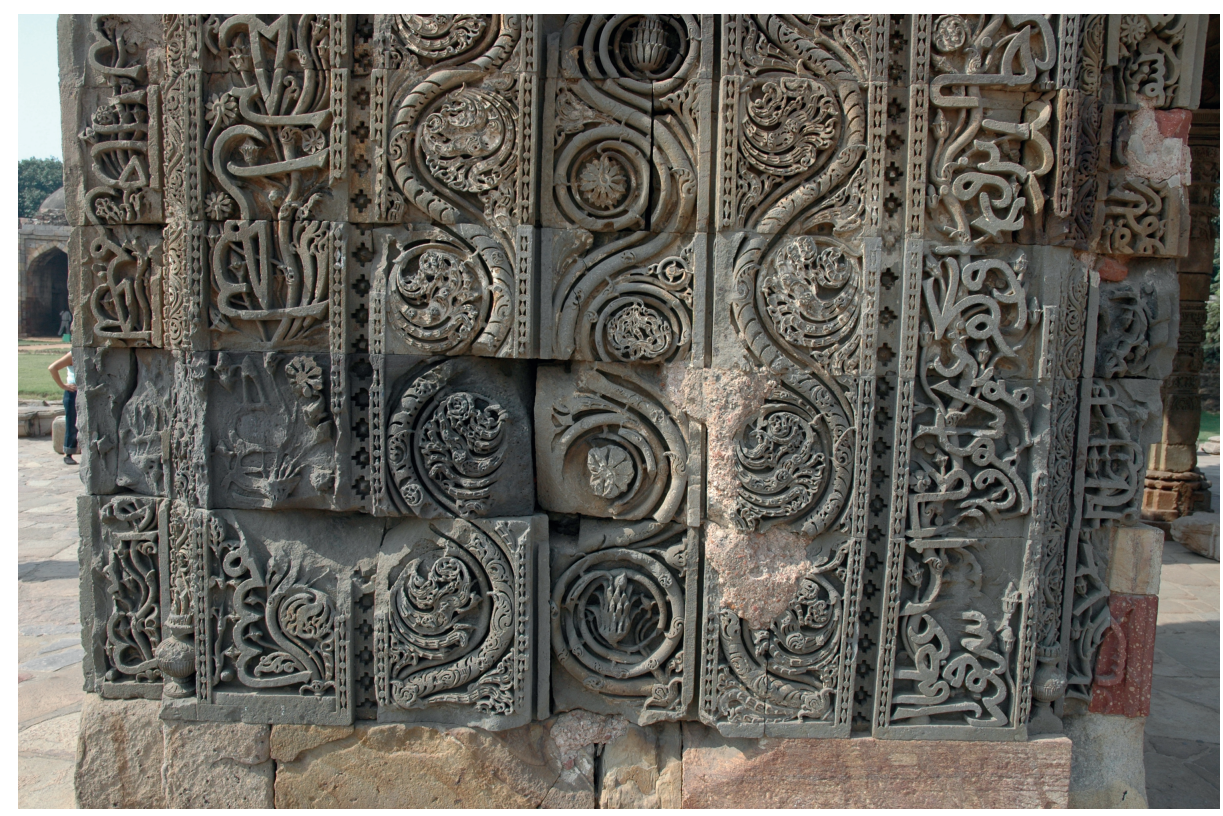

Abb. 3: Delhi, Quțb-Komplex, Detail der Blendfassade der ersten Moschee, um 1200

Sprache verfassten Texte der Inschriften auf dieser Blendfassade offenbart ein komplexeres inhaltliches Programm. Die im kursiven Duktus ausgeführten Inschriften korrespondieren mit sehr plastischen, vegetabilen und geometrischen Dekorfeldern und Bändern. Die Inschriften und Dekorbänder sind jeweils paarweise um die drei Bogenöffnungen angeordnet. Bis auf drei Inschriften sind nur zusammenhängende Versabschnitte aus dem Koran vorhanden. Die Ausnahme sind zwei hadīt-Inschriften auf den beiden inneren Inschriftenbändern um die äußeren Bogenöffnungen; auf dem zentralen Bogen gibt zudem eine unscheinbare Bauinschrift Bauherrn und Baudatum an. Jede Inschrift - einschließlich der hadìt-Inschriften auf der Blendfassade - beginnt mit der koranischen Einleitungsformel, der basmala, auch wenn diese an dieser Stelle nicht Teil des kodifizierten Korantextes ist. Die Inschriften sind dadurch auch für einen des Arabischen Unkundigen als religiöse, wenn nicht sogar koranische Texte zu erkennen. Die basmala nimmt somit - neben der Funktion als visuelle Hilfe zur inhaltlichen Einordnung der Inschriften - in Verbindung mit den trennenden Rankenbändern auf dem Blendbogen eine ähnliche Funktion an wie Koranilluminationen.

Die Auswahl der Themen in den Inschriften zeigt eine vielschichtige inhaltliche Ausrichtung. Im Zentrum des mittleren Bogens wird durch die Sure 17:1 ein Bezug $\mathrm{zu}$ wichtigen religiösen Orten des Islams, der Ka'ba in Mekka und dem Felsendom in Jerusalem, hergestellt. Die beiden hadīt-Inschriften beschreiben die Bedeutung einer Moschee für den Herrscher und die Gemeinde. Alle drei Inschriften bilden einen optisch ordnenden und thematischen Rahmen für das weitere Programm des Bogens. 
In ihm dominieren Themen wie die Beschreibung der Allmacht Gottes, seiner Schöpfung, der Offenbarung des Koran und der Zeichen, mit denen Gott sich den Gläubigen offenbart. Außerdem werden verschiedene Prophetenlegenden, wie die von Nūḥ (Noah) und Mūsā (Moses), sowie die Himmelsreise des Propheten angesprochen. Weitere Themen dieser Inschriften sind die religiösen Pflichten der Gläubigen, das Zusammenleben der Menschen und eschatologische Inhalte. Auf der Blendfassade werden also grundlegende Glaubenssätze des Islams behandelt. Erst durch horizontale und vertikale Bezüge ebenso wie durch die Hierarchisierung und Wiederholung der Inschrifteninhalte erschließt sich jedoch die über den reinen Textgehalt hinausgehende Komplexität des Programms. Beispielhaft sind aufeinander bezogene und ergänzende Textpassagen zu nennen, etwa die Beschreibungen der Höllenqualen auf dem rechten mittleren Bogen, die in den Beschreibungen der Freuden des Paradieses auf dem linken mittleren Bogen einen Gegenpart erhalten. Der innere und äußere Inschriftenbogen an einer Bogenöffnung ergänzen sich häufig gegenseitig in ihren Inhalten. Die Blendfassade wird durch eine Transformation der materiellen Präsenz des architektonischen Objektes in die Wahrnehmungs- und Erfahrungswelt des Rezipienten zu einem „Image of the Word“, ${ }^{39}$ also zum Träger einer in den Inschriften manifestierten, religiösen Glaubenswelt. Diese Glaubenswelt wird dem Gläubigen vor seinem Eintreten in den Hauptgebetsraum, ähnlich wie durch das Bildprogramm an einer hinduistischen oder jinistischen Tempelfassade, sinnbildlich ,vor Augen“ geführt.

Ein weiteres Beispiel für die Verknüpfung von textlichen Aussagen mit der hierarchischen Anordnung von Inschriften im architektonischen Raum ist an Hand der Inschriften auf dem ersten Stockwerk des sogenannten Quṭb Minārs aus der Zeit Aybaks zu beobachten (Abb. 4). ${ }^{40}$ Der Turm entstand um das Jahr 1200 und besitzt auf Grund seiner Größe, Form und technischen Realisierung eine immanente herrschaftspolitische Bedeutung. Diese findet ihren Wiederhall in den sechs Inschriftenbändern, die um das erste Stockwerk des Turms verlaufen. Auf dem untersten Inschriftenband des Turms wird der Name des Quṭb al-Dīn Aybak, des gūridischen Statthalters auf dem indischen Subkontinent, genannt, darüber sein Herr Mu'izz al-Dunyā wa al-Dīn (r. 1203-1206) und noch weiter oben in einem dritten Band der Name seines älteren Bruders Ġiyāt al-Dīn (r. 1163-1203). Die oberste Ebene nimmt ein Inschriftenband ein, das die asmā' al-ḥusnā (Gottes Schöne Namen) in der āyat al-kursī (Thronvers) der zweiten Sure enthält. Eine zusätzliche religiöse Konnotation und somit weitere Legitimität stiftende Aussagen erhalten diese persischen und arabischen Eulogien zudem durch weitere Sureninschriften in diesem Bereich in eigenen Schriftbändern oder angehängt an die genannten Bauinschriften.

39 Siehe hierzu Cruikshank Dodd 1969; Cruikshank Dodd/Khairallah 1981. 40 Page 1926, Anm. 14, 30-33. Siehe auch Pinder Wilson 2001, 171-175. 


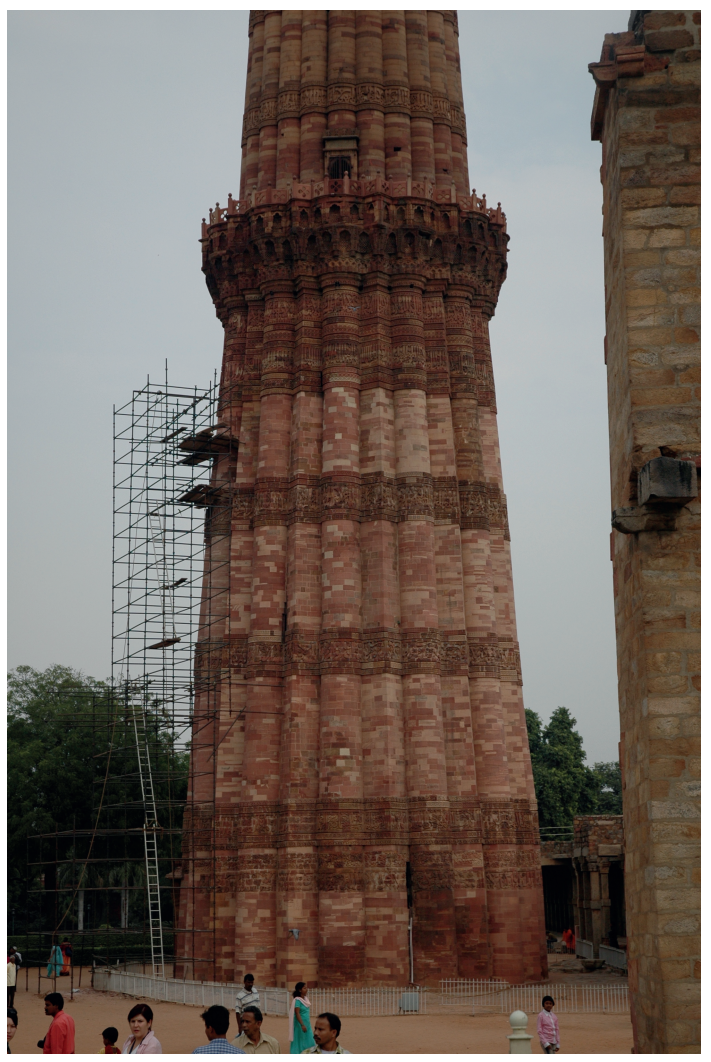

Abb. 4: Delhi, Quṭb-Komplex, Erstes Stockwerk des Quṭb Minār, um 1200

Unter der Herrschaftszeit des Iltutmiš knapp dreißig Jahre später ist eine Modifizierung der indischen Dekorelemente aus der ersten Bauphase und ihre Angleichung an einen als islamisch empfundenen Idealtypus zu beobachten. Bereits die Blendfassaden der ersten Moschee (Abb. 3) und noch deutlicher die der Erweiterung (Abb. 5) zeigen im Bereich der Flächengliederung und Dekorauswahl, wie Spitzbögen mit Bogenfeldern oder rechtwinklige Rahmen aus Inschriften und Rankensystemen, Parallelen zu Vorbildern in den islamisierten Kernländern (Abb. 6-7). In der Herrschaftszeit des Iltutmiš ist die visuelle Anknüpfung an die Bauten der Vorgänger zwar deutlich sichtbar, jedoch durchläuft der Dekor einen Prozess der Geometrisierung, Abstrahierung und Stilisierung. Diese Entwicklung setzt sich auch in der Architektur des 'Alā' al-Dīn Hilǧ̄i fort. In der Herrschaftszeit von Iltutmiš ist im Gegensatz zu der ersten Bauphase, in der fast ausschließlich auf lokale bautechnische Lösungen zurückgegriffen wird (Abb. 6), zudem ein Prozess des Experimentierens und Vermischens von regionalen und importierten Technologien $\mathrm{zu}$ beobachten. ${ }^{41}$

41 Siehe hierzu Redlinger 2008. 


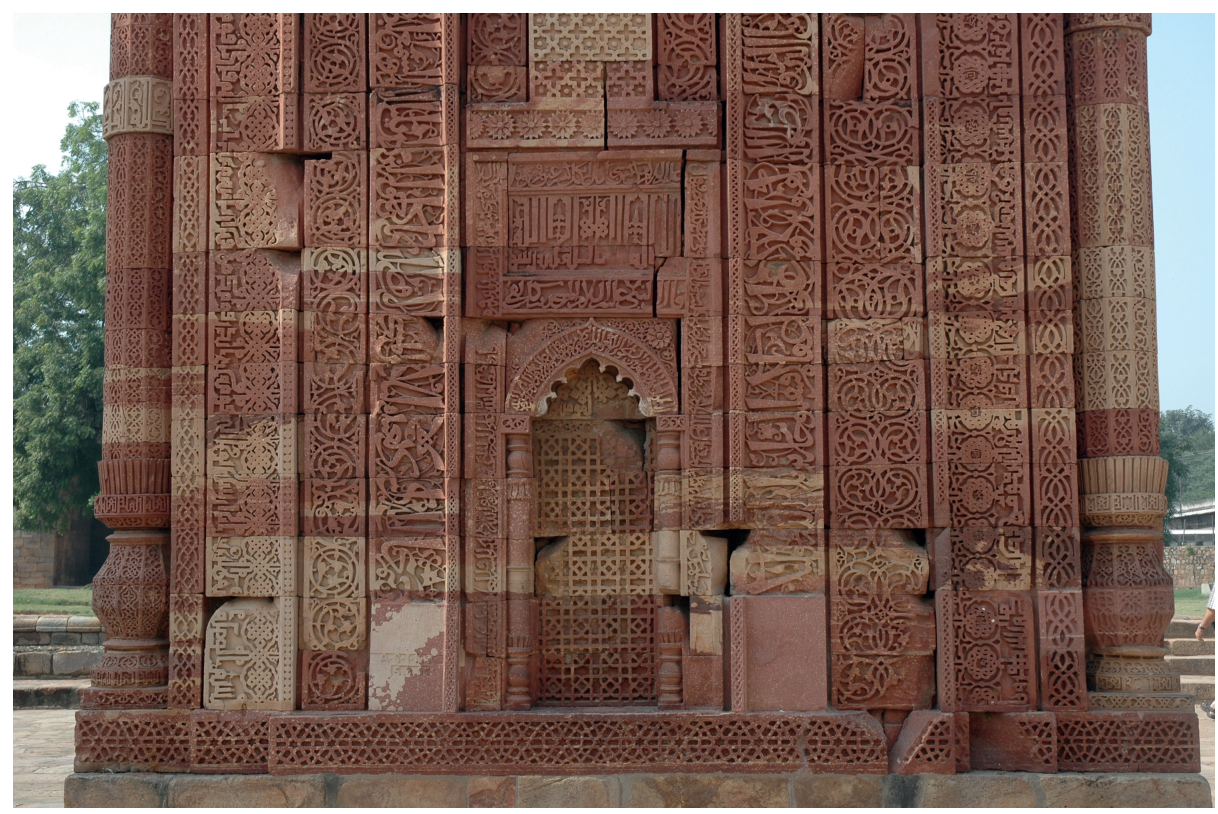

Abb. 5: Delhi, Quțb-Komplex, Quțb-Komplex, Detail der südlichen Blendfassade der ersten Erweiterung, um 1230

Der wichtigste Transformationsprozess im Rahmen der Gestaltung des ,architektonischen Textes‘ von einem durch den Dekor bestimmten ,Text‘ hin zu einem primär durch Inschriften dominierten Medium ist während Iltutmišs Herrschaft zu beobachten. In dieser Zeit werden arabische religiöse Inschriften $\mathrm{zu}$ einem bestimmenden Element der Flächengestaltung auf der Architektur, sogar weitaus mehr als geometrischer Dekor (Abb. 5-7). Erstaunlich ist, dass die zum ersten Mal auftauchenden, verschiedenen, zum Teil aufwendig gestalteten Inschriften in lapidaren und kursiven Duktus sich keinem bestimmten Themenbereich oder Texttypus zuordnen lassen. Dies zeigt, wie deutlich die arabische Schrift hier das symbolhafte Abbild des Wortes Gottes und seiner Präsenz ist. Dieser Gedanke wird gestützt durch die Beobachtung, dass alle Inschriften - außer denen aus der zweiten Bauphase des Quṭb Minār - kurze religiöse Formeln beinhalten, dem Koran oder den hadīten entnommen wurden. Eine Erklärung liegt in Iltutmišs Suche nach religiös begründeter politischer Legitimierung durch das noch existierende abbasidische Kalifat in Bagdad. Dieses Ziel erreichte er durch die offizielle Vergabe des laqab des nāșir amīr al-mu'minīn durch den Kalifen in Bagdad. Dieser für die politische und religiöse Orientierung und Herrschaftslegitimierung wichtige Ehrentitel ist dementsprechend in den Bauinschriften aus der Zeit Iltutmišs in der Architektur in Delhi und Ajmer allgegenwärtig. ${ }^{42}$

42 Siehe zur Bedeutung dieses Titels für Iltutmiš Hillenbrand 1988, Anm. 10. 
Die Bedeutungsverschiebung der Darstellung des Wortes Gottes hin zu einem Sinnbild des Glaubens zeigt sich auch darin, dass vormals durch figürliche Motive ausgedrückte Sinngehalte durch arabische Schrift ersetzt und so in eine neue visuelle Sprache übersetzt werden. Ein Beispiel sind die Zwischensteine der Säulen oder Blendsäulen (Abb. 5-7), bei denen die in der ersten Moschee - ebenso wie in der indischen Tempelarchitektur - an dieser Stelle häufig anzutreffenden kïrtimukha im Baudekor nach Aybak durch die asmā' al-ḥusnā in einem lapidaren Duktus ersetzt werden. Ein weiteres Beispiel sind Konsolensteine im Grabbau; sie haben die Form von Miniaturschreinen, jedoch ersetzen die asmá $\bar{a}^{\prime}$ al-ḥusnā in kursivem Duktus das im Kontext indischer Tempel zu erwartende Götterbild (Abb. 6).

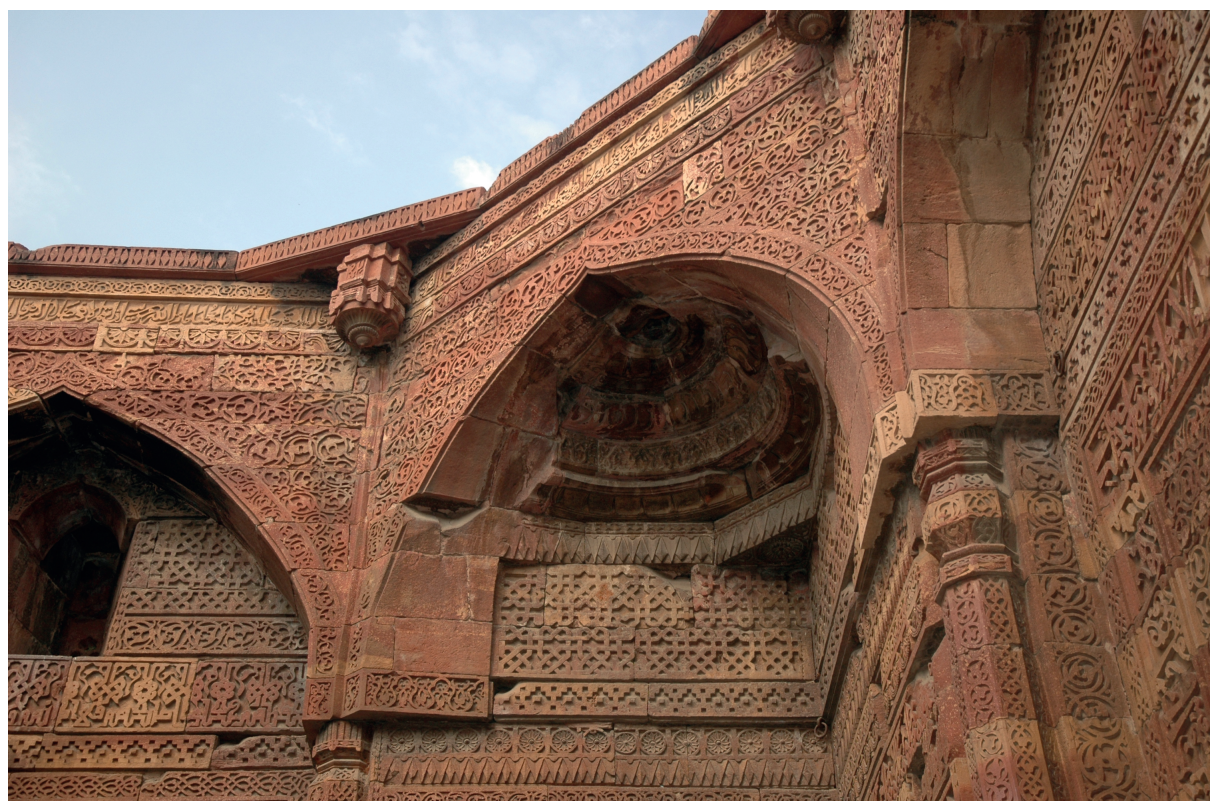

Abb. 6: Delhi, Quțb-Komplex, Detail einer Ecklösung und eines Konsolensteins im Grabbau, um 1230

Viele Inschriften, besonders im Grabbau des Iltutmiš, sind Teil der Verse, die vom Gläubigen in seinem täglichen religiösen Ritus benutzt werden. Das Inschriftenprogramm dient im Grabbau und zum großen Teil auf den Blendfassaden der Erweiterung der Moschee durch Iltutmiš, im Sinne eines aide mémoire als eine in Stein gemeißelte Unterweisung des Gläubigen über seine religiösen Pflichten (Abb.7).

Unter dem Einfluss der Emigranten aus den persischsprachigen Regionen der islamisierten Welt entwickelte sich unter 'Alā' al-Dīn Hīlğ̄i Anfang des 14. Jahrhunderts neben einem eigenständigen Bau- und Dekorkanon mit regionaler Prägung und neuen Konstruktionstechniken, wie echten Kuppeln und Trompen, auch eine neue Ausdrucksform in den Inschriften. 


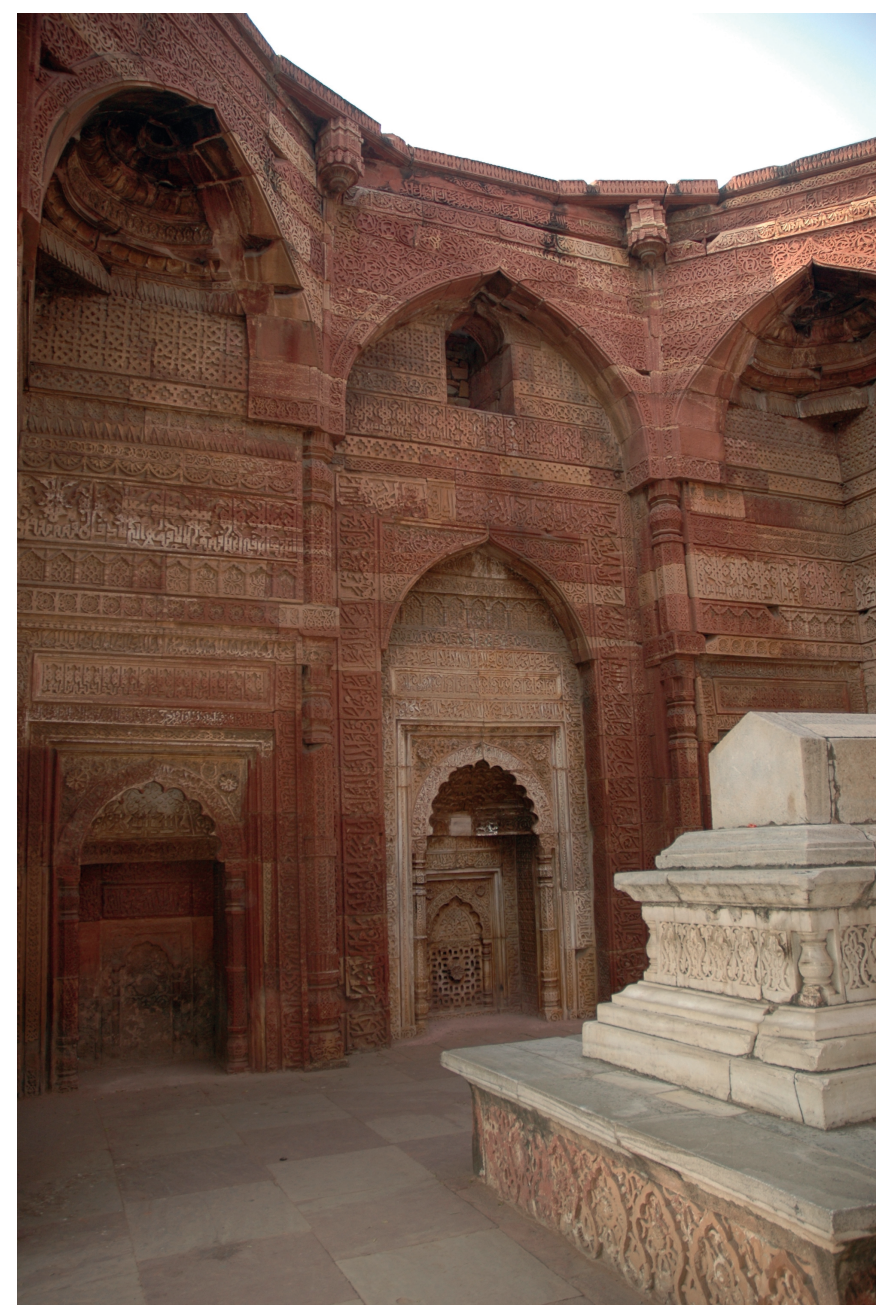

Abb. 7: Delhi, Quțb-Komplex, qibla-Wand des Grabbaus, um 1230

Die Veränderung der Architektursprache als legitimierendes Ausdrucksmittel des Herrschers lässt sich an den Bauinschriften am 'Alā'-i Darwāza aus dem Jahre 1314 nachzeichnen. Der Torbau muss, wie man aufgrund der exklusiven Baustoffe vermuten kann, eine wichtige Rolle im Hofzeremoniell gespielt haben (Abb. 8). Die Inhalte der Inschriften unterstützen diese Vermutung. Im Gegensatz zu den Bauten der Vorgänger spiegelt sich in den Inschriften aus der Herrschaftszeit 'Alā' al-Dīn Hilğìs das Selbstbild des Herrschers im Kontext eines pan-persischen, idealisierten Herrscherverständnisses wider. Die Schrift ist zu diesem Zeitpunkt deutlich formalistischer als unter Iltutmiš (Abb. 5-7) und besteht, mit Ausnahme der asmā' al-ḥusnā auf Miniatursäulen, ausschließlich aus Inschriften in kursiven Duktus (Abb. 8). Die Vielfalt des umgebenden Dekors wird drastisch reduziert. Roter Sandstein und weißer Marmor 
werden als kontraststarke Baustoffe eingeführt. Im Zentrum der drei äußeren Eingangsbereiche verlaufen um die Türöffnungen Lobpreisungen des Herrschers in persischer Sprache. ${ }^{43}$ Durch die Nennung verschiedener, mit einem perso-islamischen Geschichtsdenken verbundener Idealfiguren wird 'Alā' al-Dīn Hilğī gleichsam auf eine Ebene mit ihnen gestellt. Sowohl die Propheten als auch andere bedeutende Persönlichkeiten des Islams sind jeweils unterschiedlichen Himmelsrichtungen der Eingangsbereiche zugeordnet - so Sulaymān (Salomon) auf dem östlichen und südlichen, Darius (Dārā) und Iskandar (Alexander) auf dem östlichen und westlichen und Mūsā (Moses) auf dem südlichen Eingang. Die Nennung dieser Persönlichkeiten im Kontext eines kosmologischen und kosmographischen Konzepts und in Verbindung mit den formalen persischen Inschriften, die 'Alā' al-Dīn Huilǧì als Bewahrer und Gestalter eines islamischen Reiches und Verteidiger des Glaubens porträtieren, belegt das Ausmaß des Einflusses, den die zeitgenössische persische Schriftkunst und Geisteswelt zu diesem Zeitpunkt am Hof in Delhi erlangt hatten. Besonders die Verbindungen zum komplexen Alexanderbild und zur Figur Sulaymān (Salomon) werden hier offenkundig. ${ }^{44}$ Die wenigen Koran- und hadīt-Inschriften, die auf die eher unbedeutenden Seitenflächen beschränkt sind, dienen hier lediglich zur Verstärkung der Preisungen des Herrschers und geben ihnen einen unterstützenden legitimierenden Wert im Sinne des Islams.

Wie an Hand der Beispiele kurz skizziert wurde, stellt die Quwwat al-Islām Moschee als Schnittstelle des öffentlichen und privaten Raumes sowie Begegnungsort der muslimischen und nichtmuslimischen Bevölkerung ein wichtiges legitimierendes Ausdrucksmittel der Führungselite dar. ${ }^{45}$ Ihren die Herrschaft legitimierenden Wert erhält diese Architektur durch die Möglichkeit, in ihr spezifische Herrschaftsprogramme ,schreiben' zu können.

Einen ,Haupttext` bilden hierbei die Bauinschriften. Weitere Referenzsysteme zu identitätsstiftenden, visuellen Narrativen sind die Gestaltung der Raumhierarchien, die Verwendung von ausdrucksstarken, regional verankerten oder transregionalen Idealtypen von Bau- und Dekortechniken. Am Quṭb-Komplex lässt sich nachzeichnen, dass die Bezugnahme auf unterschiedliche Architektur- und Dekorkonzepte einem fortwährenden Transformationsprozess unterworfen war. In der ersten Bauphase unter Quṭb al-Dīn Aybak ist eine starke Relation zu lokalen Bautraditionen zu beobachten (Abb. 1-4). Dieser schließt die Einbeziehung lokaler, nichtmuslimischer Bautraditionen und Raumwirkungen wie auch den Bau auf einem prestigeträchtigen vorislamischen Bau ein. Unter Iltutmiš hingegen wurde mittels der Abstraktion und Geometrisierung lokaler Bautechniken und ästhetischer Modelle, wie auch der

43 Page 1926, Anm. 14, 34-39.

44 Zur Bedeutung des Alexanderbildes im persischsprachigen Bereich im 12.-14. Jahrhundert siehe den Konferenzband Bridges 1996.

45 Siehe zur Bedeutung der Moschee als Instrument der Herrscherlegitimation Hillenbrand 1994, 61-63. 


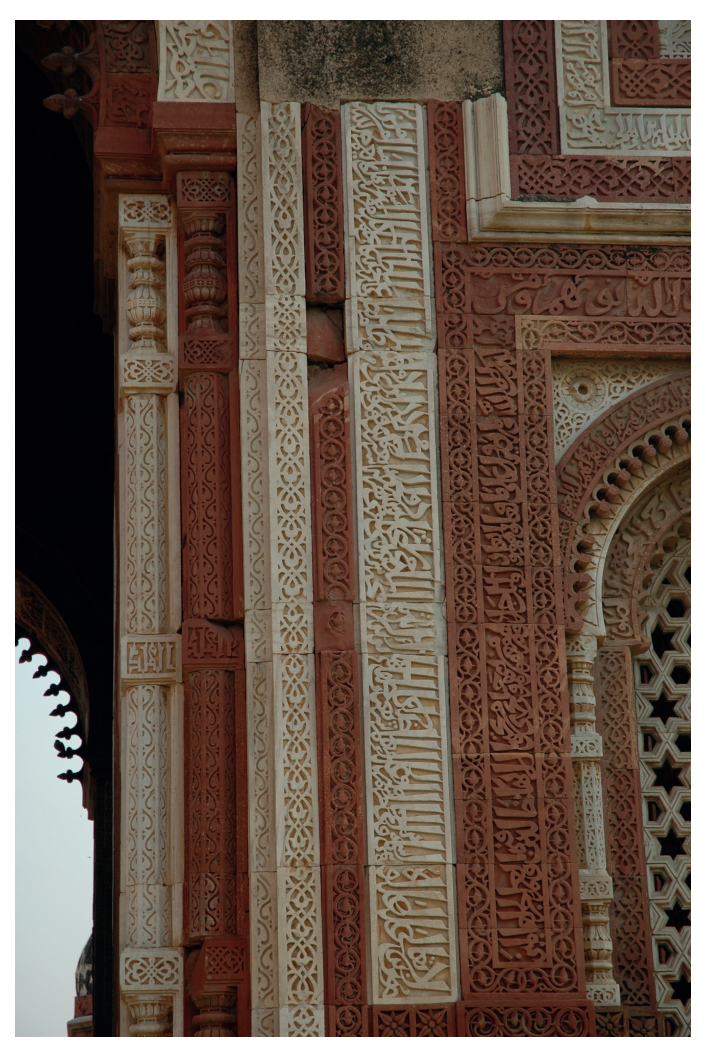

Abb. 8: Delhi, Quṭb-Komplex, westliche Außenseite des 'Alā'-i Darwāzas, um 1311

Integration von Bauelementen aus den ,islamischen Kernländern', die Architektur in eine idealtypische islamische Form umgedeutet (Abb. 5-7). Unter 'Alā' al-Dīn Hilğì gipfelte dieser architektonische Entwicklungsprozess in einen vielschichtigen indoislamischen Baukanon, der an die visuellen Erfahrungen verschiedener religiöser und sozialer Gruppe anschließen sollte (Abb. 8).

Die Bauinschriften an diesem prestigeträchtigen öffentlichen Bau übernehmen für alle drei Herrscher, als wichtiger Faktor der öffentlichen Darstellung der Sakralität von Herrschaft, maßgeblich die Funktion als Projektionsfläche für ein jeweils spezifisches politisches Programm, das mit entsprechend angepassten, identitätsstiftenden Geschichtsnarrativen verknüpft ist. Dieses Geschichtsbild ist in allen Fällen fest mit religiösen Glaubenswelten verbunden. Als das exklusivste Medium der muslimischen Glaubensgemeinschaft richten sich die Inschriften an Rezipienten, die selber den Schlüssel zum ,Lesen' und somit auch den Zugang zu den damit verbundenen Narrativen haben. Wie in der heutigen Zeit zu beobachten, erfolgte die Vermittlung sicher auch über Personen, wie imāme, šayhs oder mullās, die durch ihre höhere religiöse Ausbildung, die Texte ,übersetzen' und erläutern konnten. Die lesbaren Teile der Inschrift an der Architektur bilden somit keine rein auf den Textgehalt beschränkte 
Information, sondern dienen als Referenzsystem zu religiösen Narrativen und spezifischen idealtypischen Geschichtsbildern. So zeigt sich besonders auf der ersten Blendfassade und auf dem 'Alā'-i Darwāza, dass diese Geschichtsbilder, Wertvorstellungen und Erfahrungswerte des Islam durch Koransuren und hadīt-Inschriften transportiert werden. Durch die Erwähnung von Helden des Islams, Propheten, wichtigen Schlachten aus der Frühzeit des Islams oder eschatologischen Themen wird zusätzlich auf kodifizierte und volkstümliche Erzählungen Bezug genommen. Besonders durch die hierarchisch angeordneten Inschriften am Quṭb Minār (Abb. 4) und am 'Alā'-i Darwāza (Abb. 8) gliedern sich die Herrscher in eine spezifische, dem kollektiven Geschichtsdenken folgende Narration von einer ,Ordnung der Welt' ein. Das Beispiel am unteren Stockwerk des Turms aus der Zeit Aybaks spiegelt zudem wider, wie durch diese Narrative die Darstellung von realpolitischen Hierarchien einschließlich der Bekundungen politischer Verbindungen und Abhängigkeiten, ebenso wie auch die religiöse Legitimation abgebildet werden. Am 'Alā'-i Darwāza zeigt sich im Vergleich zu den Inschriften der Vorgängerbauten, dass sich die Bezugnahme zu Erzähltraditionen der sich wandelnden kollektiven Geschichtserfahrung anpasst.

Eine wichtige Veränderung der Übermittlung von in der Architektur verankerten Narrativen ist auch bei der Transformation vom Bild zu einem ,Text-Bild' ab der zweiten Bauphase zu erkennen. Wie an den Zwischensteinen der Säulen (Abb. 5, 7-8), und an den Konsolensteinen im Innern des Grabbaus besonders deutlich wird (Abb. 6), werden die lokalen nichtmuslimischen Bildtraditionen direkt durch das Wort Gottes in arabischer Schrift übersetzt.

Die Beispiele zeigen, dass dem Rezipienten durch ein komplexes Verweissystem anhand der Inschriften und ihrer visuellen und gegebenenfalls akustischen Präsentation mittels der Rezitation oder Erläuterung der Inhalte durch einen Kundigen vor Ort umfangreiche Narrative nahegebracht werden. Vergleichbar sind diese durch die Inschriften vermittelten ,Bilder im Kopf‘ mit dem Bilddekor in Tempeln, der den Gläubigen ihre Glaubenswelt vor Augen führt, eine Orientierung in dieser vermittelt und den religiösen Ritus kontextualisiert. Inschriften bilden in der Quwwat al-Islām Moschee somit ein mnemotechnisches System, das die lokale Bildtradition durch eine neue visualisierte Form der Geschichtserzählung, quasi in Form einer ,Schriftbildlichkeit‘, ersetzt.

Wie am Beispiel des Quṭb-Komplexes gezeigt werden kann, dienen die Bauinschriften in der Herrschaftsarchitektur dazu, die Deutungshoheit über identitätsstiftende kohärente Geschichtsnarrative zu erlangen. Dies schließt die Transformation von dekorativen Formen als Teil eines komplexen visuellen Referenzsystems für historische Erinnerungen und ästhetische Erfahrung mit ein. Identitätsstiftende und legitimierende Geschichte wird somit von den unterschiedlichen Herrschern, angepasst an ihre jeweiligen Herrschaftsprogramme, ähnlich einem dreidimensionalen Film als visualisierter und sinnlich wahrnehmbarer ,historischer Raum` in die Architektur projiziert. 


\section{Literaturverzeichnis}

Belting, Hans (2006), Bild-Anthropologie. Entwürfe für eine Bildwissenschaft, München.

Belting, Hans (2012), Florenz und Bagdad. Eine westöstliche Geschichte des Blicks, München.

Berkey, Jonathan (1992), The Transmission of Knowledge in Medieval Cairo, Princeton.

Blair, Sheila S. (2006), Islamic Calligraphy, Edinburgh.

Bridges, Margaret (Hg.) (1996), The Problematics of Power. Eastern and Western Representations of Alexander the Great, Bern.

Cole, Henry H. (1872), The Architecture of Ancient Delhi, specially the Buildings around the Qutb Minar, London.

Conermann, Stephan (2002), Indo-persische Geschichtsschreibung während der Mogulzeit (932-1118/1516-1707) (Historiographie als Sinnstiftung 5), Wiesbaden.

Cruikshank Dodd, Erica (1969), „The Image of the Word. Notes on the Religious Iconography of Islam“, in: Berythus 18, 35-62.

Cruikshank Dodd, Erica/Khairallah, Shereen (1981), The Image of the Word. A Study of Quranic Verses in Islamic Architecture, Beirut.

Cunningham, Alexander/Beglar, J. D./Carlleyle, A. C. L. (1874), Archaeological Survey of India: Reports for the Year 1871/72, Calcutta.

Eaton, Richard M. (2000), „Temple Desecration and Indo-Muslim States“, in: David Gilmartin u. Bruce B. Lawrence (Hgg.), Beyond Turk and Hindu. Rethinking Religious Identities in Islamicate South Asia, Gainesville, 246-281.

Flood, Finbarr B. (2001), „Ghūrid Architecture in the Indus Valley. The Tomb of Shaykh Sādan Shahīd“, in: Ars Orientalis 31, 129-166.

Flood, Finbarr B. (2003), „Pillars, Palimpsests, and Princely Practices. Translating the Past in Sultanate Delhi“, in: Research Anthropology and Aesthetics 43, 95-116.

Flood, Finbarr B. (2007), „Lost in Translation. Architecture, Taxonomy, and the Eastern ,Turks““, in: Muqarnas: An Annual on Islamic Art and Architecture 24, 79-115.

Flood, Finbarr B. (2008), „The Origins of the Qutb Mosque and Qutb Minar. A Nineteenth-Century Controversy, Debate between Alexander Cunningham and J. D. Beglar", in: Finbarr B. Flood (Hg.), Piety and Politics in the Early Indian Mosque, New Delhi, 97-119.

Flood, Finbarr B. (2009), Objects of Translation. Material Culture and Medieval „Hindu-Muslim“ Encounter, Princeton (NJ)/Oxford.

Gomolla, Stephanie (2002), „Lesbare Architektur und architektonischer Text. Metaphern und deren Überwindung bei Michel Butor“, http://www.metaphorik.de/02/gomolla.htm (Stand 23.04.2013).

Grabar, Oleg (1992), The mediation of ornament. The A.W. Mellon lectures in the fine arts 1989, Princeton (NJ).

Grabar, Oleg (2006), „What Makes Islamic Art Islamic?“, in: Oleg Grabar (Hg.), Islamic Art and Beyond (Constructing the Study of Islamic Art 3), Hampshire, 245-252.

al-Harithy, Howayda (2010), „Inscriptions and the Making of Public Space in Mamluk Cairo“, in: Huda Smitshuijzen AbiFarès (Hg.), Typographic matchmaking in the city: propositions for a pluralistic public space, Amsterdam, 18-29.

Hilgert, Markus (2010), „,Text-Anthropologie‘: Die Erforschung von Materialität und Präsenz des Geschriebenen als hermeneutische Strategie“, in: Markus Hilgert (Hg.), Altorientalistik im 21. Jahrhundert. Selbstverständnis, Herausforderungen, Ziele (Mitteilungen der Deutschen Orient-Gesellschaft 142), 87-126.

Hillenbrand, Robert (1988), „Political Symbolism in Early Indo-Islamic Mosque Architecture. The Case of Ajmīr“, in: Iran 26, 105-117.

Hillenbrand, Robert (1994), Islamic Architecture. Form, Function, and Meaning, Edinburgh. 
Husain, Maulvi Muhammad Ashraf (1936), Records of All the Quranic and Non-Historical Epigraphs on the Protected Monuments in the Delhi Province (Memoirs of the Archaeological Survey of India 47), Calcutta.

Jackson, Peter (1990), „The ,Mamlūk“ Institution in Early Muslim India“, in: Journal of the Royal Asiatic Society of Great Britain and Ireland 2, 340-358.

Jackson, Peter (1999), The Delhi Sultanate. A Political and Military History, Cambridge/New York. Juvin, Carine (2013), „Mamluk Inscriptions“, in: Stephan Conermann (Hg.), Ubi Sumus? Quo Vademus? Mamluk Studies, State of the Art (Mamluk Studies 3), Göttingen, 211-229.

Krämer, Sybille (2003), „>Schriftbildlichkeit< oder: Über eine (fast) vergessene Dimension der Schrift“, in: Sybille Krämer u. Horst Bredekamp (Hgg.), Bild, Schrift, Zahl, München, 157-176.

Kumar, Sunil (2008), „Quțb and Modern Memory“, in: Finbarr B. Flood (Hg.), Piety and Politics in the Early Indian Mosque, New Delhi, 150-178.

Kumar, Sunil (2009), „The Ignored Elites. Turks, Mongols and a Persian Secretarial Class in the Early Delhi Sultanate“, in: Modern Asian Studies 43/1, 45-77.

Kumar, Sunil (2010), The Emergence of the Delhi Sultanate, 1192-1286, Ranikhet/Bangalore.

Lermer, Andrea/Shalem, Avinoam (Hgg.) (2010), After One Hundred Years. The 1910 Exhibition „Meisterwerke muhammedanischer Kunst“ Reconsidered (Islamic History and Civilization 82), Leiden /Boston.

Mujeeb, Mohammad (2008), „The Qutub Complex as a Social Document“, in: Finbarr B. Flood (Hg.), Piety and Politics in the Early Indian Mosque, New Delhi, 120-129.

Niazi, Ghulam Sarwar Khan (1990), The Live and Works of Sultan Alaudin Khalji, Lahore.

Page, James A. (1926), An Historical Memoir on the Qutb, Delhi (Memoirs of the Archaeological Survey of India 22), Calcutta.

0’Sullivan, Shaun (2006), „Coptic Conversion and the Islamization of Egypt“, in: Mamluk Studies Review 10 (2), 65-79.

Patel, Alka (2004), „Toward Alternative Receptions of Ghurid Architecture in North India (Late Twelfth-Early Thirteenth Century (E)“, in: Archives of Asian Art 54, 35-36.

Patel, Alka (2004a), Building Communities in Gujarāt. Architecture and Society during the Twelfth through Fourteenth Centuries (Brill's Indological Library 22), Leiden/Boston (MA).

Patel, Alka (2004b), „Communities and Commodities. Western India and the Indian Ocean, EleventhFifteenth Centuries", in: Ars Orientalis 34, 7-18.

Patel, Alka (2009), „Recasting the Architectural Landscape. The Late 12th-Early 13th Century Ghurid Annexations of Northern India“, in: Gerd J. R. Mevissen u. Arundhati Banerji (Hgg.), Prajñâdhara. Essays on Asian Art, History, Epigraphy and Culture in Honour of Gouriswar Bhattacharya, New Delhi, 122-136.

Pinder Wilson, Ralph (2001), „Ghaznavid and Ghūrid Minarets“, in: Iran 39, 155-186.

Redlinger, Daniel (2008), „Die frühe Islamische Architektur Indiens als Medium eines programmatisch formulierten Herrschaftsanspruchs. Die Quwwat al-Islām Moschee in Delhi“, in: Berichte über die Tagung in Bamberg vom 1. bis 2. Juli 2005 und in Bonn vom 7. bis 8. Juli 2006 (Beiträge zur Islamischen Kunst und Archäologie 1), Wiesbaden, 145-160.

Redlinger, Daniel (2012), Architektur, Dekor und Inschriftensysteme am Quțb Komplex in Delhi. Architektur als Kulisse und Quelle für historische Konstrukte (unpubl. Diss. Islamwissenschaft, Universität Bonn).

Redlinger, Daniel (2012a), „Kulturwissenschaften. Die Antworten der Orientalischen Kunstgeschichte“, in: Stephan Conermann (Hg.), Was ist Kulturwissenschaft? Zehn Antworten aus den „Kleinen Fächern“, Bielefeld, 199-232.

Sanderson, G. (1916), Archaeological Works at the Qutb 1912-1913 (Archaeological Survey of India Annual Reports, 1912-1913), Calcutta. 
Shokoohy, Mehrdad (1988), Bhadreśvar. The Oldest Islamic Monuments in India (Studies in Islamic Art and Architecture 2), Leiden/New York.

Shokoohy, Mehrdad (1993), Architecture of the Muslim Trading Communities in India (Beiträge zur Südostasienforschung Südasien-Institut, Universität Heidelberg: Islam and Indian Religions 145), Heidelberg.

Shokoohy, Mehrdad (2003), Muslim Architecture of South India. The Sultanate of Ma'bar and the Traditions of the Maritime Settlers on the Malabar and Coromandel Coasts (Tamil Nadu, Kerala and Goa), London/New York.

Tabbaa, Yasser (2001), The transformation of Islamic art during the Sunni revival, Seattle.

Welch, Anthony (1977), „Epigraphs and Icons. The role of the writing“, in: Joseph Gutmann (Hg.), The image and the word. Confrontations in Judaism, Christianity and Islam, Missoula, 63-74.

Welch, Anthony (1985), „Qur’ān and Tomb. The Religious Epigraphs of Two Early Sultanate Tombs in Delhi“, in: Frederick M. Asher u. G. S. Gai (Hgg.), Epigraphy. Its Bearing on the History of Art, Delhi, 257-267.

Welch, Anthony/Keshani, Hussein/Bain, Alexandra (2002), „Epigraphs, Scripture, and Architecture in the Early Delhi Sultanate“, in: Muqarnas. An Annual on Islamic Art and Architecture 19, 12-43.

Wink, André (1997), „The Slave Kings and the Islamic Conquest. 11th-13th Centuries“, in: André Wink (Hg.), Al-Hind. The Making of the Indo-Islamic World. Bd. 2, Leiden.

\section{Abbildungsverzeichnis}

Abb. 1: Delhi, Quțb-Komplex, Nordkolonnade der ersten Moschee, um 1200 (Foto: Redlinger).

Abb. 2: Delhi, Quțb-Komplex, Bauinschrift im Osteingang, um 1200 (Foto: Redlinger).

Abb. 3: Delhi, Quțb-Komplex, Detail der Blendfassade der ersten Moschee, um 1200 (Foto:

Redlinger).

Abb. 4: Delhi, Quțb-Komplex, Erstes Stockwerk des Quțb Minār, um 1200 (Foto: Redlinger).

Abb. 5: Delhi, Quṭb-Komplex, Detail der südlichen Blendfassade der ersten Erweiterung, um 1230

(Foto: Redlinger).

Abb. 6: Delhi, Quțb-Komplex, Detail einer Ecklösung und eines Konsolensteins im Grabbau, um 1230

(Foto: Redlinger).

Abb. 7: Delhi, Quțb-Komplex, qibla-Wand des Grabbaus, um 1230 (Foto: Redlinger).

Abb. 8: Delhi, Quṭb-Komplex, westliche Außenseite des 'Ală'-i Darwasas, um 1311 (Foto: Redlinger). 
